Michael Beißwenger (Duisburg-Essen)

\title{
Internetbasierte Kommunikation als Textformen-basierte Interaktion: ein neuer Vorschlag zu einem alten Problem
}

\begin{abstract}
Der Beitrag behandelt die Frage, wie sich das spezifisch Neue internetbasierter Kommunikation unter linguistischer Perspektive fassen und in Traditionen des sprachlichen Handelns einordnen lässt. Es wird gezeigt, dass sich die internetbasierte Kommunikation weder als Interaktion noch als Textkommunikation hinreichend beschreiben lässt, zugleich aber Merkmale mit beiden Formen teilt. Mit dem Konzept der Textformen-basierten Interaktion wird ein Vorschlag formuliert, wie dieses Dilemma aufgelöst werden kann: Das Innovationspotenzial internetbasierter Kommunikationstechnologien liegt gerade darin, dass diese durch Indienstnahme von Textformen die Möglichkeiten von Interaktion erweitern. Das hat einerseits Konsequenzen für die linguistische Analyse und lässt sich andererseits fruchtbar machen für die Entwicklung von Standards für die Repräsentation von Korpora.
\end{abstract}

\section{Einleitung}

Im Kontext der Modellierung und Analyse sprachlicher und kommunikativer Praktiken bildet die internetbasierte Kommunikation einen spannenden Untersuchungsgegenstand. Geradezu Fishbowl-artig lässt sich an (und in) ihr studieren, wie sich die Nutzerinnen und Nutzer internetbasierter Kommunikationstechnologien an die von der Technologie gesetzten Rahmenbedingungen anpassen, um unter diesen Bedingungen bestmöglich das zu tun, wozu die Technologie gemacht ist: Interaktion zu gestalten.

Die Kommunikation im Netz ist dabei ständigem technologischem Wandel unterworfen. Anwendungen verändern sich: Facebook und Twitter weisen heute ein anderes Funktionsspektrum auf als vor fünf Jahren. Die Kommunikationsform Chat feiert seit dem Aufkommen mobiler Messenger-Anwendungen wie WhatsApp ein Revival als Instrument für die digital vermittelte, sequenziell organisierte Individualkommunikation. Nutzerinnen und Nutzern, die über diese Anwendungen kommunizieren und ihre sozialen Netzwerke pflegen, stehen damit andere 
Ressourcen zur Verfügung als in früheren Versionen desselben Systems oder in Vorläufersystemen; entsprechend ändern sich auch die Praktiken der sozialen Vernetzung und der Interaktionsgestaltung. Der Forschungsgegenstand ,Kommunikation in Social-Media-Umgebungen' ist unter dieser Perspektive schwierig zu fixieren. Er ist ein moving target. Die nachhaltige Präsentation datengestützter linguistischer Untersuchungen zum Gegenstand ist daher in der Regel mit einem nicht unerheblichen Dokumentationsaufwand verbunden, insofern die technischen Bedingungen, unter denen Sprachdaten produziert wurden, auf Stand des Produktionszeitpunkts erfasst und als äußere Rahmenbedingungen für den Nachvollzug der an ihnen analytisch rekonstruierten Praktiken beschrieben werden müssen.

Bei all der Variabilität von Ressourcen und darauf bezogenen Praktiken gibt es aber durchaus Formate, die sich als sehr beständig gegenüber dem stetigen technologischen Wandel erwiesen haben, auch wenn sie uns in einzelnen Apps und Plattformen in unterschiedlichen Instanziierungen gegenübertreten. Die Tatsache, dass diese Formate übergreifend zu einzelnen Softwareanwendungen und -versionen in verschiedensten Kommunikationstechnologien immer wieder neu reproduziert werden, darf als Reflex der technologischen Entwicklung auf Nutzungspraktiken und die in diesen Praktiken artikulierten Bedarfe der Nutzerinnen und Nutzer aufgefasst werden: Die durch Software bereitgestellten Funktionalitäten werden durch massenhafte und wiederholte Nutzung in unterschiedlichsten Kontexten und für unterschiedliche Zwecke über soziale Praxis validiert; die damit an die Entwicklerinnen und Entwickler rückgespiegelte Akzeptanz führt dazu, dass bewährte Funktionskonstellationen bei der Weiterentwicklung der Technologien beibehalten werden.

In der Präsentation und im exakten Funktionsumfang unterschiedlich, hinsichtlich zentraler Strukturmerkmale aber von hoher Übereinstimmung sind beispielsweise die folgenden Formen internetbasierter Kommunikation: Threads auf Diskussionsseiten der Wikipedia; Sequenzen von aufeinander bezogenen Kommentar-Postings zu YouTube-Videos; Diskussionsverläufe in Online-Foren, deren Beiträge in chronologischer oder thematischer Anordnung am Bildschirm persistent vorgehalten werden; ein Tweet mit den dazu verfassten und am Bildschirm darunter angeordneten Antwort-Tweets; initiale Postings auf Facebook-Seiten mit den dazu verfassten Folgebeiträgen anderer Nutzerinnen und Nutzer; Chatverläufe auf WhatsApp oder in Webchats, in denen die Beiträge der einzelnen Beteiligten in zeitlicher Abfolge auf einem prinzipiell endlosen Protokoll vorgehalten werden. Auf den ersten Blick mögen in diesen Formen internetbasierter Kommunikation zunächst die Unterschiede ins Auge stechen. In struktureller Hinsicht weisen die Kommunikationsverläufe - sowohl als Produkte als auch im Prozess ihrer Hervorbringung - aber dieselbe grundlegende Merkmalhaftigkeit auf, auch 
wenn die einzelnen Umgebungen ihren Nutzerinnen und Nutzern ganz unterschiedliche Gestaltungsmöglichkeiten für ihre Kommunikationsbeiträge bereitstellen und die ausgetauschten Äußerungen in jeweils unterschiedlicher Optik am Bildschirm präsentieren.

20 Jahre, nachdem das Leibniz-Institut für Deutsche Sprache (IDS) erstmals eine Jahrestagung dem Thema „Sprache und neue Medien“ gewidmet hat (1999), ist die Kommunikation im Internet „reif“ genug, um danach $\mathrm{zu}$ fragen, wie sich die in der Zwischenzeit etablierten Formate - als gesellschaftlich verifizierte und in Software kondensierte Erfolgsmodelle - in ihrer Spezifik beschreiben lassen. Für ein umfassendes Verständnis des „Phänomens Internet“ ist nicht nur die Analyse der jeweils neuesten Entwicklungen von Interesse, sondern auch, unter all der Dynamik und Vielfalt nach sich abzeichnender Kontinuität zu forschen.

Ein solcher Versuch soll mit dem vorliegenden Beitrag unternommen werden. Ausgehend von den Gemeinsamkeiten, die prominente Formen internetbasierter Kommunikation aufweisen, widmet er sich der Frage, wie sich diese Gemeinsamkeiten in Form eines Basisformats internetbasierter Kommunikation beschreiben lassen. Die folgenden Fragestellungen lege ich dabei zugrunde:

1. Was ist das spezifische „Neue“ an der internetbasierten Kommunikation? Diese Frage ist ebenso alt wie die linguistische Beschäftigung mit dem Gegenstand. Sie zieht sich durch die Forschungsliteratur wie ein roter Faden - bis heute.

2. Wie lässt sich ein Basisformat internetbasierter Kommunikation beschreiben, das deutlich macht, in welchen Traditionen die dialogisch-sequenziell organisierte Kommunikation in Chats, Foren, WhatsApp, auf Wikipedia-Diskussionsseiten, auf Facebook und auf Twitter steht? Hier interessieren mich die Gemeinsamkeiten mit und die Unterschiede zu Organisationsformen sprachlichen Handelns, für die die Linguistik bereits über etablierte Beschreibungsmodelle verfügt - die Interaktion und das sprachliche Handeln mit Texten.

In Hinblick auf das Basisformat, für dessen Spezifik ich ein Beschreibungsmodell vorlegen werde, konzentriere ich mich auf Kommunikationsformen wie die oben aufgeführten. Entsprechend verwende ich im Weiteren den Terminus ,Internetbasierte Kommunikation“ in diesem eingeschränkten Sinne. Ich schließe nicht aus, dass es ggf. mehrere Basisformate internetbasierter Kommunikation gibt. Das Format, um dessen Rekonstruktion es mir geht, ist dennoch nicht zufällig herausgegriffen. Es begegnet uns in der Geschichte internetbasierter Kommunikationsformen seit Jahrzehnten sehr prominent und in einer großen Zahl von Anwendungen und ist bereits in frühen Vorläuferformen heutiger Chat-, Foren- und Messaging-Anwendungen aus den 1980er-Jahren belegt. Damals war es aufgrund begrenzter Übertragungsbandbreiten undenkbar, gesprochene Sprache zeitnah über das Internet zu vermitteln. Entsprechend blieb als alternative Möglichkeit 
für die Realisierung eines dialogisch-sequenziell organisierten, zeitnahen Austauschs nur die Schrift, und zwar in einer auf das Zeicheninventar des ASCII-Codes reduzierten Version. Neben (kurzlebigen) Systemen mit zeichenweiser Übermittlung (UNIX Talk) setzte sich dabei schon früh ein Format durch, bei dem Zeichenketten paketiert übermittelt wurden (vgl. Beißwenger 2007, S. 64-66) - passend zu dem auf die paketierte Datenübertragung ausgelegten TCP/IP-Protokollsockel des Internets. Die Entscheidung für Schriftlichkeit ist somit ursprünglich technologiegeschichtlich begründet und sozusagen aus der Not geboren - mit weitreichenden Konsequenzen für den gesellschaftlichen Umgang mit Schrift und für den dadurch ausgelösten sprachlichen Wandel (vgl. dazu Storrer 2014).

Ich greife im Folgenden zunächst exemplarisch einige Positionen aus der linguistischen Diskussion auf, die ich für die Entwicklung des Ausgangspunkts meiner Argumentation benötige (Kap. 2). Davon ausgehend werde ich einen Modellierungsvorschlag für das angezielte Basisformat entwickeln, der sich als Beitrag zur linguistischen Diskussion versteht (Kap. 3 und 4). Abschließend werde ich zeigen, wie dieser Vorschlag auch für die Repräsentation von Korpora fruchtbar gemacht werden kann und im Bereich der Digital Humanities bereits konkret Anwendung findet (Kap. 5).

\section{Einige Positionen aus der linguistischen Diskussion}

Die Frage „Wie kann ein geeignetes Modell zur Erfassung der Spezifik internetbasierter Kommunikation aussehen?“ ist eng verknüpft mit der Frage, welche Modelle für die Organisation sprachlichen Handelns, die die Linguistik bereits ausgearbeitet hat, sich sinnvoll auf die Beschreibung des Gegenstands übertragen lassen. Dass die internetbasierte Kommunikation mit Blick auf ihre im Grundsatz sequenziell intendierte Organisationsform der Interaktion näher steht als dem Text, kann mit Blick auf die Forschungsliteratur als weitgehend unstrittig gelten. Dürscheid/Brommer (2009) und Imo (2013, S. 281) kommen zu dem Schluss, dass es zwar durchaus Unterschiede zu mündlichen Interaktionen gebe, die Gemeinsamkeiten aber überwögen; entsprechend sei der Analysezugang der Interaktionalen Linguistik (Selting/Couper-Kuhlen 2000) grundsätzlich auch auf die internetbasierte Kommunikation anwendbar - wenngleich, wie in der frühen, konversationsanalytisch ausgerichteten Forschung zur computer-mediated communication wiederholt gezeigt wurde, Analysekategorien, die an mündlichen Gesprächen entwickelt wurden, bei der Anwendung auf schriftlich realisierte Formen auf den Prüfstand gestellt und ggf. angepasst werden müssen. Eine viel- 
zitierte Arbeit ist in diesem Zusammenhang Garcia/Baker Jacobs (1999). Die Autorinnen weisen auf den grundsätzlich asynchronen Charakter internetbasierter Kommunikation hin, und zwar selbst in Fällen wie den klassischen Chats, in denen die Beteiligten zeitlich kopräsent sind. Garcia und Baker Jacobs prägen in diesem Zusammenhang den Ausdruck ,quasi-synchronous computer-mediated communication‘ (ebd., S. 339), einen Terminus, der in der Folge neben anderen von Dürscheid (2005) aufgegriffen wird.

Zitzen/Stein (2005, ähnlich auch Beißwenger 2003), führen konkreter aus, worin diese Asynchronie besteht, und verweisen auf die grundsätzliche Entkoppelung der Produktions- von der Rezeptionssituation. Insbesondere beim Chatten hat das erhebliche Auswirkungen auf das Interaktionsmanagement, wie in Beißwenger (2007) empirisch gezeigt wird. Zitzen/Stein (2005) kommen anhand ihrer Befunde zu dem Vorschlag, neben orality und literality für die internetbasierte Kommunikation ein Drittes anzunehmen, das sie vorläufig als digitality bezeichnen (ebd., S. 1015f.). Wiewohl viele der Beobachtungen und Feststellungen in der Arbeit von Zitzen und Stein sehr treffend sind, hat sich dieser Vorschlag nicht durchgesetzt, was vermutlich daran liegt, dass ein Konzept ,konzeptionelle Digitalität‘ (digitality) in seiner Beziehung zur Mündlichkeit und Schriftlichkeit sowie $\mathrm{zu}$ den damit in Zusammenhang stehenden Traditionen $\mathrm{zu}$ unspezifisch und damit forschungspraktisch kaum handhabbar ist.

Es lohnt sich aber durchaus darüber nachzudenken, ob die internetbasierte Kommunikation nicht „ein Drittes“ darstellt - und zwar nicht ein Drittes neben Mündlichkeit und Schriftlichkeit, sondern vielmehr ein Drittes neben Interaktion und Text. Angelika Storrer spricht in ihrem Beitrag zum IDS-Jahrbuch 2013 - dort in Bezug auf Korpora internetbasierter Kommunikation - von Korpora „dritter Art“ (Storrer 2014, S. 189-191), deren Gegenstand sich von mündlichen Gesprächen wie auch von schriftlichen Texten so weitgehend unterscheide, dass für seine Erfassung neue Methoden und Werkzeuge benötigt würden. Zugleich zeigt Storrer, dass die sprachliche Gestaltung internetbasierter Kommunikation in Traditionen des Schreibens steht - das aber im Falle von Chat, Foren und vergleichbaren Formen den Zwecken der Interaktion folgt. Entsprechend führt Storrer (2013, 2014) das Konzept des ,interaktionsorientierten Schreibens' in die Diskussion ein, das es erlaubt, die Spezifik der sprachlichen Gestaltung schriftlicher internetbasierter Kommunikation gegenüber der sprachlichen Gestaltung von Texten nicht nur auf der Ebene einzelner sprachlicher Phänomene zu beschreiben, sondern auch konzeptuell zu profilieren.

Mit den Arbeiten von Imo/Lanwer (2019) und Hausendorf et al. (2015) soll im Folgenden noch auf zwei Positionen eingegangen werden, die die Frage, ob die internetbasierte Kommunikation sinnvoll als ein Fall von Interaktion zu beschreiben ist, auf sehr unterschiedliche Weise beantworten. 
Imo/Lanwer (2019) entwickeln im Rahmen des Ansatzes der Interaktionalen Linguistik die Perspektive einer „Interaktionalen Schriftlinguistik“ und führen an Analysen vor, wie sich die Konzepte der interaktionalen Linguistik auf die Kommunikation in WhatsApp anwenden lassen. Zugleich machen sie deutlich, dass die Übertragung des Ansatzes auf schriftlich realisierte Kommunikation in theoretischer und methodischer Hinsicht noch weiter entwickelt werden müsse:

Mit zunehmenden Forschungsergebnissen [ist] auch kritisch zu evaluieren, welche Konzepte aus der Konversationsanalyse und der Interaktionalen Linguistik für die Analyse von interaktionaler Schriftlichkeit übernommen werden können, welche angepasst werden müssen und wo man eventuell vollständig neue Konzepte benötigt. (Imo/Lanwer 2019, S. 294)

Hausendorf et al. (2015) gehen demgegenüber von einem Interaktionskonzept aus, das Interaktion für die Kommunikation unter Anwesenden reserviert, und betonen - wo Imo/Lanwer die Gemeinsamkeiten herausstellen - gerade die Differenz der schriftlichen Kommunikation im Netz zur Face-to-face-Begegnung: Für sie wird in der internetbasierten Kommunikation Anwesenheit durch Lesbarkeit ersetzt. Entsprechend beschreiben sie die internetbasierte Kommunikation als eine Form der „Textkommunikation, [die] in den Dienst eines dialogartigen Austauschs gestellt“ wird (Hausendorf et al. 2015, S. 126). Die beiden Perspektiven gegenübergestellt demonstrieren sehr eindrücklich, wie neu und wie schwierig zu fassen der Gegenstand für die Linguistik nach wie vor ist. Beide Vorschläge haben Erklärungskraft, akzentuieren die Stellung der internetbasierten Kommunikation zwischen Interaktion und Text aber ganz unterschiedlich. Gerade die Differenz zwischen den beiden Vorschlägen erscheint mir als sehr produktiv.

\section{Internetbasierte Kommunikation als ein Drittes}

\subsection{Internetbasierte Kommunikation als Weiterentwicklung vorgängiger Organisationsformen des sprachlichen Handelns}

Die Spezifik internetbasierter Kommunikation ist meines Erachtens weder als „Interaktion mit Abstrichen“ noch als „Textkommunikation mit gewissen Besonderheiten“ zufriedenstellend erfasst. In Anbetracht der bedeutenden Rolle, die netzbasierte Formen der Kommunikation in immer mehr Bereichen des Alltags einnehmen, erachte ich es für nicht mehr zeitgemäß, der internetbasierten Kom- 
munikation, obwohl deren Besonderheiten in der Forschung fraglos erkannt sind, nach wie vor nur einen Quasi-Status zuzuweisen. Wenig informativ und forschungspraktisch kaum operationalisierbar ist auch ein Label wie „Digitalität“ zur Beschreibung des Besonderen internetbasierter Kommunikation gegenüber mündlichen Gesprächen und schriftlichen Texten. Sinnvoll erscheint mir vielmehr eine Sichtweise, die die internetbasierte Kommunikation als ein Drittes auf einem Entwicklungsweg von Organisationsformen des sprachlichen Handelns betrachtet, an dessen Beginn (1) die mündliche Interaktion steht, dessen zweite Stufe (2) die Indienstnahme von Schriftlichkeit und Textformen und die gesellschaftliche Aneignung der situationsentbundenen Kommunikation mit Texten darstellt, und auf dem sich, ermöglicht durch die Kommunikationsinfrastruktur des Internets, (3) eine dritte Organisationsform ergeben hat, die die Qualitäten von Textformen adaptiert, um situationsentbundene, sequenziell intendierte Kommunikation zu ermöglichen. ${ }^{1}$

Die Perspektive auf den Gegenstand, die ich damit entwickeln möchte, kann als Vorschlag aufgefasst werden, die Idee einer ,Interaktionalen Schriftlinguistik ${ }^{*}$ im Sinne von Imo/Lanwer (2019) zu präzisieren und weiterzuentwickeln. ${ }^{2}$ Zugleich betone ich damit aber auch die neuen, ganz und gar Interaktions-untypischen Möglichkeiten der Gestaltung und Organisation kommunikativen Austauschs, die dann erkennbar werden, wenn man die ausgetauschten Produkte und die Strukturen, in die sie eingebettet sind, unter dem Gesichtspunkt ihrer Textformenhaftigkeit (und nicht nur ihrer medialen Schriftlichkeit) betrachtet.

Damit greife ich einen Gedanken von Hoffmann (2004) auf, der die „Nutzung der Qualität von Textformen“ für die Organisation sequenziell intendierter Kommunikation als zentrales Charakteristikum internetbasierter Kommunikation beschrieben hat. In Beißwenger (2007) habe ich diesen Gedanken weiterentwickelt und die strukturelle Differenz der Chat-Kommunikation zum Gespräch empirisch ausgeleuchtet. Darauf aufbauend habe ich in Beißwenger (2016) eine Perspektive für

1 Situationsentbundene Kommunikation wird hier als Kommunikation unter Bedingungen der Zerdehnung (im Sinne von Ehlich 1983) aufgefasst, also als Kommunikation, in der der Kontakt zwischen den Beteiligten nicht über ein gemeinsames Hier und Jetzt gestiftet wird.

2 Eine solche Weiterentwicklung wäre freilich mit der Anforderung verbunden, Textformen und Schreiben als konstitutive Ressourcen der Interaktionsgestaltung systematisch mitzudenken und den methodischen Zugriff auf den Gegenstand entsprechend zu adaptieren. Das schließt ein, von gegenüber dem mündlichen Gespräch fundamental unterschiedlichen Zeitlichkeitsbedingungen auszugehen und Konzepte der Interaktionsanalyse, die auf die Zeitlichkeitsbedingungen gesprochener Sprache bezogen sind, auf den Prüfstand zu stellen und - ggf. auch rigoros - für die neuen Rahmenbedingungen anzupassen. 
die Analyse von Praktiken in der internetbasierten Kommunikation entwickelt, die die Zeitlichkeitsbedingungen von Kommunikation als den Dreh- und Angelpunkt betrachtet, um Organisationsformen sprachlichen Handelns in ihrer Spezifik zu erfassen und miteinander zu vergleichen. An diese Überlegungen knüpfe ich im Folgenden an.

Unter einer Textform verstehe ich in Anlehnung an Hoffmann (2004) eine Repräsentationsform für sprachliche Äußerungen, die der Äußerung Überlieferungsqualität verleiht, was die Organisation sprachlichen Handelns unter Bedingungen der ,Zerdehnung (sensu Ehlich 1983, 1984 et passim) ermöglicht. Die Überwindung von Flüchtigkeit (durch Ermöglichung von Persistenz) ist dafür konstitutives Merkmal. In Kombination mit schriftlicher Realisierung stellen Textformen weitere Qualitäten für die Gestaltung sprachlichen Handelns bereit: Flächigkeit, Visualität, multimodale Gestaltbarkeit. Von diesen Merkmalen und den Möglichkeiten ihrer Pragmatisierung wird in Kapitel 4 die Rede sein.

Die auf Stufe (2) des oben skizzierten Entwicklungswegs über Jahrhunderte ausgearbeitete gesellschaftliche Institution ,Text' stellt die prototypische Form der Indienstnahme von Textformen für Zwecke der Kommunikation unter Distanzbedingungen (zeitlich, räumlich, sozial) dar. Der Rückgriff auf Textformen ist aber nicht auf die Kommunikation unter Distanzbedingungen beschränkt. Zettelkommunikation wäre ein Beispiel ohne technische Vermittlung, das allerdings als gesellschaftlich randständig gelten kann und nur unter speziellen Kontextbedingungen auftritt. Erst die technologischen Innovationen des Internets stellen die Rahmenbedingungen und Potenziale bereit, unter denen Textformen und Schrift im großen Stil Aufgaben bei der Organisation sequenziell intendierter Kommunikation im Nahbereich übernehmen können. Die dadurch ausgelöste systematische Nutzung der Schrift im Nahbereich führt zu sprachlichen Besonderheiten, deren Auftreten Storrer (2014) als Indiz für sprachliche Ausbauprozesse (extensiv, intensiv) beschrieben hat.

\subsection{Zeitlichkeitsbedingungen von Gesprächen - Zeitlichkeitsbedingungen internetbasierter Kommunikation}

Konstitutives Merkmal von Textformen ist die Überwindung von Flüchtigkeit. Dieses Merkmal kann in seiner Leistung und in seinen Folgen für die Organisation und Gestaltung sprachlichen Handelns nicht hoch genug eingeschätzt werden. Für die Analyse der Zeitlichkeitsbedingungen internetbasierter Kommunikation 
möchte ich im Folgenden beim mündlichen Gespräch ansetzen, an dessen Kommunikationsbedingungen die Kategorien einer auf die Spezifik des Interaktionalen ausgerichteten Sprachanalyse entwickelt wurden. Vor diesem Hintergrund lässt sich entwickeln, wie fundamental sich die Textformen-basierte Interaktion von Gesprächen unterscheidet.

Eine Analyse von Praktiken der gesprochenen Sprache ist ohne Berücksichtigung ihrer Zeitlichkeitsbedingungen und der daraus erwachsenden Ressourcen nicht denkbar (vgl. Auer 2000, 2007; Deppermann 2007; Hausendorf 2007; Couper-Kuhlen 2007 u.a.). Zeitlichkeitsbedingungen sind in der Mündlichkeit wesentlich auf die Dispositionen und auf die Reichweite körperlicher Mittel bezogen, die Deppermann (2018, S. 52) als „Infrastruktur leiblichen Handelns“ beschreibt.

Aus der Körpergebundenheit lassen sich zentrale Zeitlichkeitsbedingungen mündlicher Gespräche ableiten: die Flüchtigkeit und die Irreversibilität der Verbalisierung, die Synchronisierung der „Bewusstseinsströme des Ich und des Du“ (Auer 2000, S. 46), auf deren Hintergrund Sprachstrukturen als grundsätzlich interaktional und kollaborativ erzeugte Leistungen zu konzipieren sind (Selting/ Couper-Kuhlen 2000, S. 81), sowie die Emergenz und die Inkrementalität des Strukturaufbaus, die sich auf allen Ebenen der sprachlichen Gestaltung und Organisation von Gesprächen auswirken.

Während die Zeitlichkeitsbedingungen mündlicher Gespräche an eine Infrastruktur der Leiblichkeit geknüpft sind, ist im Falle technisch vermittelter Interaktion Sprache eingebettet in eine Infrastruktur technischer Vermittlung, bei der - sensu Weingarten (1989, S. 32) - der ,ganzheitliche, kontinuierliche und variable Kommunikationsprozeß [...] nach festen Regeln in Ebenen und Einheiten zerlegt und so für den technischen Prozeß zugänglich gemacht“ wird und für die charakteristisch ist, dass gerade nicht unmittelbar leiblich gehandelt werden kann.

Im Fall der internetbasierten Kommunikation hat das zur Konsequenz, dass die Zeitlichkeitsbedingungen mündlicher Gespräche nicht gelten. Wir betrachten ein Datenbeispiel aus dem Dortmunder Chat-Korpus, das bereits in Beißwenger (2005, S. 84 f.) und Beißwenger (2007, S. 268 f.) analysiert wurde und das in der Folge Imo (2013, S. $278 \mathrm{f}$.) noch einmal interaktional-linguistisch interpretiert hat (Abb. 1). An diesem Beispiel lässt sich sehr gut zeigen, wie die interaktionale Perspektive mit einer Perspektive der Textformenhaftigkeit zusammengebracht werden kann (bzw. muss), um der Spezifik der Interaktionskonstitution in Abhängigkeit von den technologisch gesetzten Rahmenbedingungen gerecht $\mathrm{zu}$ werden. Wir sehen einen Ausschnitt aus einem chatbasierten Beratungsangebot. BER ist der Berater, RS ein Ratsuchender. 


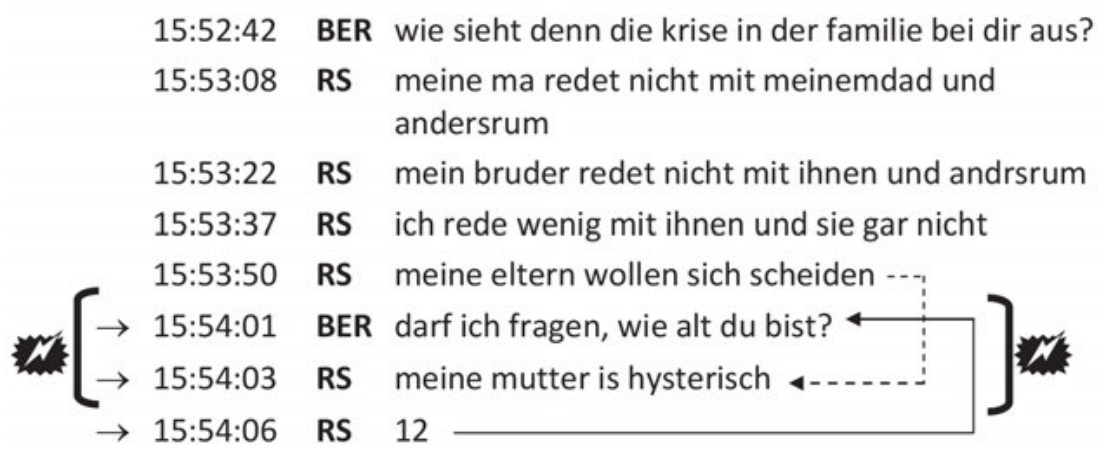

Abb. 1: Ausschnitt aus einem Chatverlauf

Auf den ersten Blick zeigt das Beispiel in Abbildung 1 eine sequenziell strukturierte Folge von Beiträgen, die mit klaren „Sprecherwechseln“ aufeinander folgen. Bei näherer Betrachtung knirscht es aber im sequenziellen Gebälk: Die Platzierung der einzelnen Äußerungen gelingt den Beteiligten nicht in jeder Hinsicht so wie es sequenziell Sinn ergeben würde: Auf die Frage von BER „darf ich fragen, wie alt du bist" folgt mit nur zwei Sekunden Versatz als vermeintliche Antwort von RS die Äußerung „meine mutter is hysterisch“. Tatsächlich handelt es sich hier nicht um die Antwort auf die unmittelbar davor angezeigte Frage, sondern um die Fortsetzung einer davor über mehrere Postings verteilten, längeren Äußerung, mit der RS seine familiäre Situation schildert. BER hat möglicherweise die Äußerung von RS mit dem Beitrag 15:53:50 als abgeschlossen interpretiert und daraufhin eine Folgefrage produziert, während RS - für BER unsichtbar - mit der Produktion einer weiteren Fortsetzung seiner Äußerung beschäftigt war. Dass BER zwischenzeitlich eine neue Frage gestellt und einen Themenwechsel eingeleitet hat, hat RS vermutlich erst nach der Versendung seines Beitrags bemerkt. Er schiebt daraufhin eine weitere Äußerung hinterher - „12“-, die als Antwort auf die zuvor gestellte Frage passt.

Als Resultat ergeben sich, wenn man nur das Verlaufsprotokoll in die Analyse einbezieht, Sequenzmuster, die für mündliche Gespräche eher untypisch wären - von Garcia/Baker Jacobs (1999) als ,Phantomadjazenz', von Imo (2015, S. 23) als ,sequenzielle Überlappung charakterisiert. Diese Interpretationen sind unter interaktionaler Perspektive naheliegend, bleiben aber oberflächennah. Dass die Beteiligten einen sequenziell organisierten Austausch führen möchten, ist unstrittig. Dass sie für dessen Strukturierung auf Konzepte zurückgreifen, die ihnen aus mündlichen Gesprächen vertraut sind, auch. $\mathrm{Zu}$ differenzieren ist aber zwischen (1) der Sequenzplanung der einzelnen Interaktionsbeteiligten, (2) der Umsetzung dieser Planung in schriftliche Beiträge, (3) der Einordnung der versen- 
deten Beiträge in das linear strukturierte Verlaufsprotokoll am Bildschirm und (4) der jeweils individuellen Wahrnehmung dieses aktuellen Stands. Durch die beständige Anforderung, Lese- und Schreibaktivitäten individuell zu koordinieren, kann nicht davon ausgegangen werden, dass es zu einem Zeitpunkt eine geteilte Auffassung der Beteiligten über den Stand des Interaktionsgeschehens gibt. Der Standardfall ist die Divergenz der individuellen Sichten auf den Stand der Interaktion. Der zeitliche Versatz mag zwar in vielen Fällen gering sein; die grundsätzliche Entkopplung von Produktion, Übermittlung und Rezeption führt aber dazu, dass die Sequenzorganisation nicht über ein kollaboratives Interaktionsmanagement zur Laufzeit erzeugt werden kann. Kohärente Anschließbarkeit muss vielmehr individuell hergestellt werden, in der Produktion und in der Rezeption.

Die Zeitlichkeitsbedingungen der internetbasierten Kommunikation gleichen unter dieser Perspektive viel eher den Zeitlichkeitsbedingungen beim sprachlichen Handeln mit Texten als in mündlichen Gesprächen. Frank Liedtke hat in seinem Beitrag „Schrift und Zeit“ (2009) drei Zeitpunkte unterschieden, die für das Handeln mit Texten konstitutiv sind: einen Inskriptionszeitpunkt, zu welchem die Äußerung produzierendenseitig vollständig ausformuliert und verschriftet vorliegt; einen Rezeptionszeitpunkt, zu welchem die Textäußerung von einem Rezipienten oder einer Rezipientin gelesen und damit rezipientenseitig aktualisiert wird; einen Obligationszeitpunkt, zu welchem als Resultat der Lektüre die „Festlegungen, Berechtigungen und Verpflichtungen“ (Liedtke 2009, S. 80) der mit dem Text intendierten sprachlichen Handlungen realisiert sind. Der Charakter der zeitlichen Ruptur beim sprachlichen Handeln mit Texten - die ,Zerdehnung sensu Ehlich $(1983,1984)$ - lässt sich allerdings noch detaillierter erfassen: Dem von Liedtke beschriebenen Inskriptionszeitpunkt lässt sich ein Intentionszeitpunkt voranstellen, zu welchem der bzw. die Produzierende beschließt, eine Textäußerung zu konzipieren. Der Intentionszeitpunkt bildet den Ausgangspunkt für die Produktion; der Zeitraum zwischen Intentions- und Inskriptionszeitpunkt bildet die Produktionszeit. Vom Inskriptionszeitpunkt ist weiterhin ein Verfügbarkeitszeitpunkt zu unterscheiden, der denjenigen Zeitpunkt bezeichnet, ab welchem die inskribierte Textäußerung für die Rezipientin oder den Rezipienten - typischerweise nach einem vorangegangenen Übermittlungs- oder Publikationsprozess zugänglich ist. Der Verfügbarkeitszeitpunkt fällt nicht notwendigerweise zusammen mit dem Zeitpunkt der Wahrnehmung der Textäußerung durch potenzielle Rezipierende; zugleich ist der Zeitpunkt der Wahrnehmung der Textäußerung nicht identisch mit dem Zeitpunkt der Obligation, da für die rezipierendenseitige Aktualisierung der mit dem Text vollzogenen Handlungen zunächst die Rezeption erforderlich ist, die als Zeitraum zwischen einem Perzeptionszeitpunkt und dem bereits charakterisierten Obligationszeitpunkt beschrieben werden kann. Insgesamt ergeben sich, um die spezifischen Zeitlichkeitsbedingungen für die 
Konstitution des sprachlichen Handelns mit Texten in einem Modell zu erfassen, fünf relevante Zeitpunkte. Diese sind in Abbildung 2 veranschaulicht.

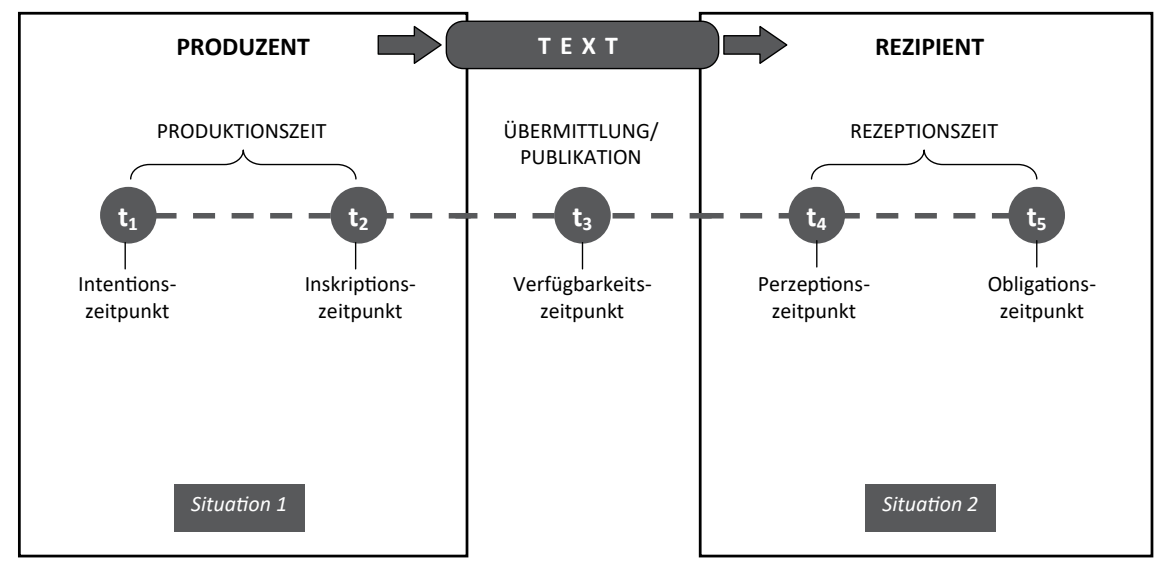

Abb. 2: Zeitlichkeitsbedingungen beim sprachlichen Handeln mit Texten

Für die internetbasierte Kommunikation muss dieses Modell nur geringfügig angepasst werden (Abb. 3). Inskribiert ist die Äußerung zunächst bei der bzw. dem Produzierenden. Nach der Übermittlung erscheint sie im Verlaufsprotokoll auf dem Endgerät der Adressatin bzw. des Adressaten. Zwischen dem Intentionszeitpunkt und der Verarbeitung des Beitrags durch die Adressaten können sich die Kontextbedingungen für die mit dem Beitrag intendierten sprachlichen Handlungen jederzeit ändern - weil sich das Bildschirmprotokoll ändern kann. Wie ein Beitrag sequenziell in den Kontext „passt“, lässt sich für die oder den Produzierende/n erst nach der Übermittlung und Platzierung im Protokoll, genau genommen sogar erst an den Folgebeiträgen der Rezipient/innen, ablesen. Das Verlaufsprotokoll am Bildschirm bildet dabei die zentrale Ressource, um sequenzielle Zuordnungen in der Rezeption herzustellen bzw. ggf. auch erst im Nachhinein $\mathrm{zu}$ rekonstruieren.

Am Beispiel aus Abbildung 1 lässt sich das zeigen: Die sinnvolle sequenzielle Zuordnung des Beitrags „meine mutter is hysterisch“ bedarf für BER des Rücksprungs auf einen früheren Stand als denjenigen, den er mit seiner Frage „darf ich fragen, wie alt du bist?“ erzeugt hat. RS kann die gestellte Frage - obwohl in der Anzeige ein sequenziell deplatziertes Element interveniert - nachträglich beantworten, ohne das Thema der Frage sprachlich erneut aufgreifen zu müssen. Die Frage ist schriftlich am Bildschirm vorgehalten, und er kann seinem Partner unterstellen, dass dieser in der Lage ist, den entsprechenden Anknüpfungspunkt 
im Protokoll eigenaktiv zu identifizieren. Die Persistenz des Geäußerten im Protokoll leistet somit einen wesentlichen Beitrag, um die gegenüber mündlichen Gesprächen eingeschränkten Möglichkeiten des Interaktionsmanagements zur Laufzeit bearbeitbar zu machen.

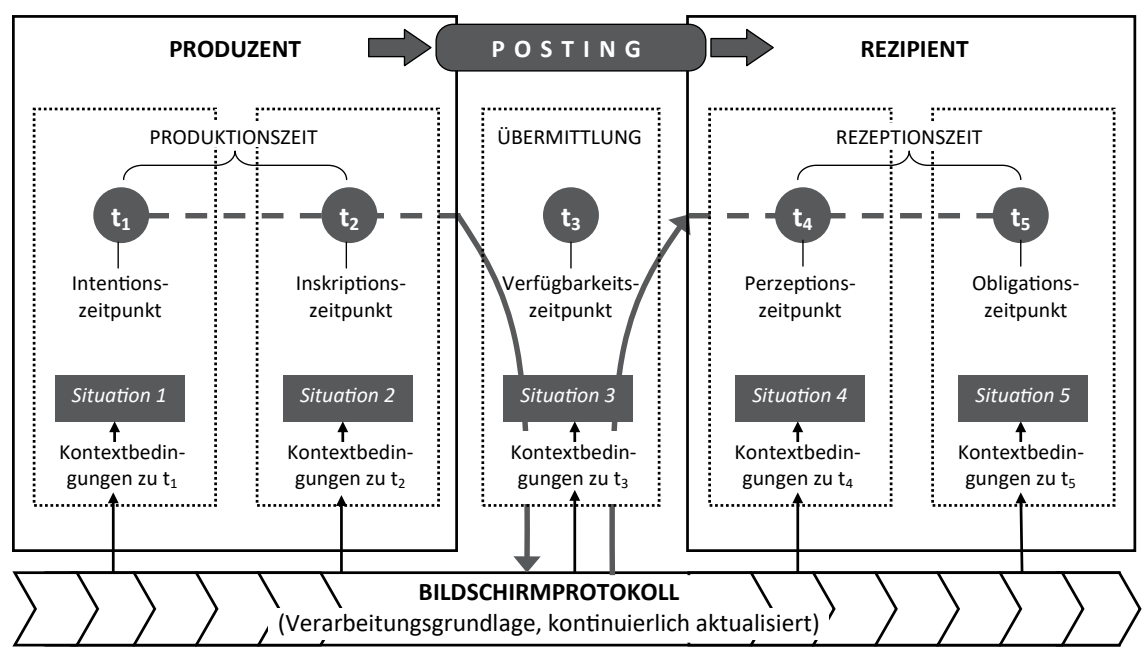

Abb. 3: Zeitlichkeitsbedingungen in der internetbasierten Kommunikation (vgl. Beißwenger 2007, 2016)

Persistenz ist eine Ressource nicht nur auf der Ebene des Verlaufsprotokolls, sondern auch auf der Ebene der Produktion. Eingegebene Entwürfe werden dadurch wiederlesbar, editierbar und reversibel. Der Prozess der Formulierung und Gestaltung schriftlicher Beiträge kann damit rekursiv und reflexiv gestaltet werden analog zu monologischen Schreibprozessen, bei denen der aufgeschriebene Textentwurf vom Schreibenden zum Input für den weiteren Schreibprozess und damit zum Gegenstand der Evaluation, der Reflexion und der erneuten Bearbeitung werden kann. Dass diese Möglichkeit auch tatsächlich und systematisch genutzt wird, habe ich in Beißwenger (2007) empirisch an einer Analyse von Prozessen der Beitragsproduktion in Chats gezeigt.

\subsection{Zwischenfazit: Textformen-basierte Interaktion}

Die Ausgangsfragen aus Kapitel 2 lassen sich auf dem Hintergrund der in den Abschnitten 3.1 und 3.2 präsentierten Analyse der Zeitlichkeitsbedingungen inter- 
netbasierter Kommunikation wie folgt beantworten: In der internetbasierten Kommunikation ist, technologiegeschichtlich bedingt und eher zufällig, etwas Neues, Drittes, entstanden, das sich aber als enorm erfolgreich erwiesen hat. Die internetbasierte Kommunikation ist Textformen-basierte Interaktion und als solche eine Weiterentwicklung vorgängiger Organisationsformen sprachlichen Handelns - der Interaktion und des Textes - mit spezifisch eigenen Organisationsprinzipien und Leistungen. Systematisch werden in ihr Textformen für die Organisation eines als interaktional intendierten Austauschs genutzt. Textformen machen Potenziale der Verdauerung sprachlicher Information als Ressource für die Organisation von Kommunikation verfügbar und ermöglichen die zeitliche Entkopplung von Produktion, Übermittlung und Rezeption. Dialogisch-sequenziell intendierter Austausch wird dadurch zeitlich flexibel gestaltbar; die sequenziellen Zuschreibungen entstehen nicht als unmittelbar interaktionale Leistungen der Beteiligten, sondern in der individuellen Handlungsplanung und Rezeption. Dadurch bleibt eine grundsätzliche Differenz sowohl zur Interaktion als auch zum Text. Deshalb ist die Textformen-basierte Interaktion ein Drittes - gerade weil sie in Traditionen sowohl der Interaktion als auch des sprachlichen Handelns mit Texten steht. In den klassischen Chats hat sie ihr volles Potenzial nicht ausspielen können; vermutlich deshalb spielen klassische Chats, bei denen alle Beteiligten zeitgleich online sein mussten, heute nur noch eine randständige Rolle. Die Weiterentwicklung der klassischen Chats zu Anwendungen wie WhatsApp mit sitzungsübergreifend vorgehaltenem Verlaufsprotokoll „entschärft“ das Problem der Sequenzorganisation gerade dadurch, dass Interaktion zeitflexibler gestaltbar wird. Hinzu treten vielfältige Möglichkeiten der multimodalen Gestaltung von Kommunikaten, von denen in Kapitel 4 noch zu sprechen sein wird.

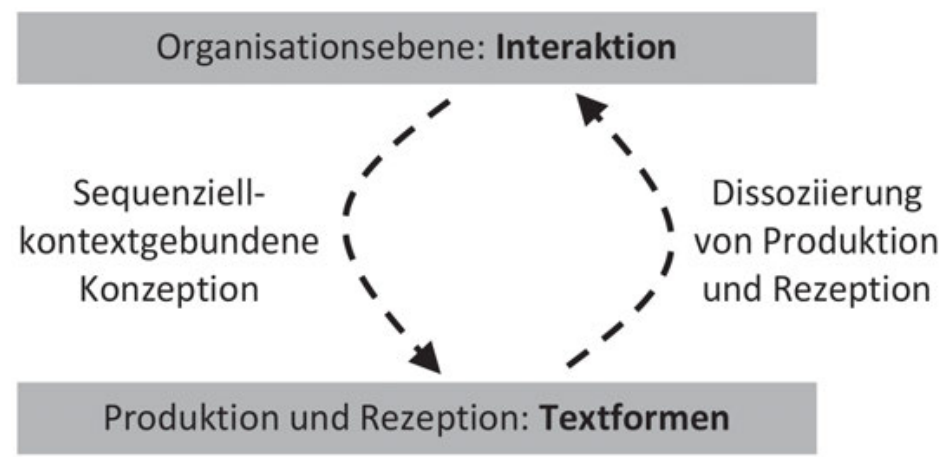

Abb. 4: Textformen-basierte Interaktion 
Zwei Arten von Textformen sind prägend für das Basisformat internetbasierter Kommunikation, um das es mir geht:

1. das Posting: eine Zeichenfolge, die en bloc verschickt, nach Aufbereitung durch das vermittelnde System als flächiges Ganzes am Bildschirm präsentiert und dort visuell gegen Einheiten gleichen Typs abgegrenzt wird (Beißwenger 2016, 2018);

2. das Verlaufsprotokoll am Bildschirm, das die Verarbeitungsgrundlage bildet und in dem die Postings als Resultat des Zusammenspiels von Nutzereingaben und Systemroutinen in eine lineare, ggf. auch in Threads verzweigende Ordnung gebracht und in dieser Ordnung für die wiederholte Rezeption vorgehalten werden.

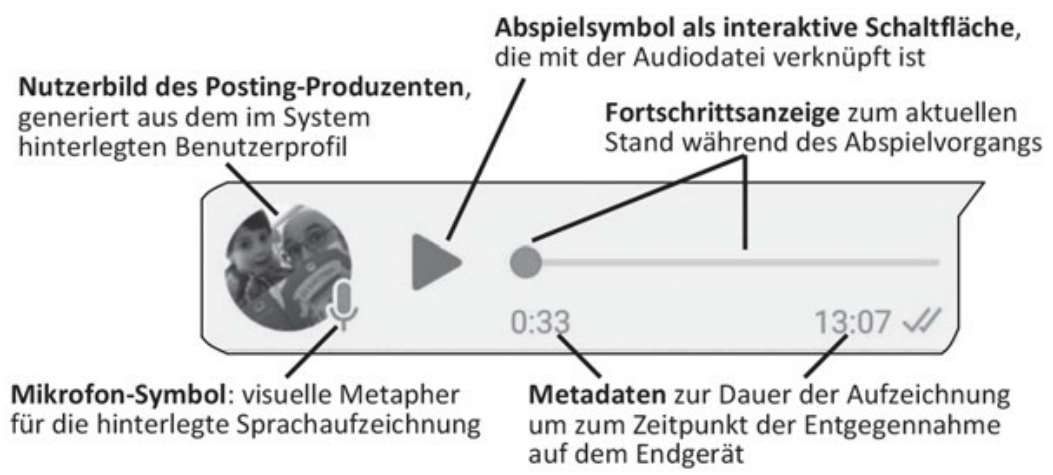

Abb. 5: Präsentation eines Audio-Postings am Bildschirm als visuelle, flächige, multimodale Form

Dass sich die Textformen-basierte Kommunikation als Erfolgsmodell entpuppt hat, zeigt sich einerseits in ihrer massenhaften Verbreitung, andererseits aber auch an der noch relativ jungen Innovation der sogenannten „Sprachnachrichten“ (Abb. 5). Sprachnachrichten ermöglichen es den Nutzerinnen und Nutzern von WhatsApp, eine Audio-Datei zu besprechen und über die Chatfunktion zu versenden. Sehr treffend sprechen Katharina König und Tim Moritz Hector (2017) in Bezug auf Sprachnachrichten von Audio-Postings. Mit Audio-Postings „hält die mediale Mündlichkeit mit all ihren Ausdrucksressourcen Einzug in die mobile MessengerKommunikation“ (König/Hector 2017, S. 36). Das Inventar der verfügbaren Gestaltungsmittel wird dadurch erweitert, Chats können wahlweise oder alternierend medial mündlich und medial schriftlich organisiert werden. Selbst bei ausschließlicher Nutzung von Sprachnachrichten bleibt der Chat aber strukturell Textformenbasiert; eine Annäherung an Gesprächsformen findet lediglich auf der Ebene der medialen Realisierung als gesprochene Sprache statt - aber selbst das unter dem 
Vorbehalt, dass zentrale Zeitlichkeitsbedingungen mündlicher Gespräche nicht gelten. Dazu passt auch, dass Audio-Postings im Verlaufsprotokoll als visuelle, flächige, multimodale Formen präsentiert werden, über die mittels Aktivierung eines Abspielsymbols die Audio-Aufzeichnung abgerufen werden kann.

\section{Textformen-basierte Interaktion als Kommunikation über multimodale Sehflächen}

An der Gestaltung von Audio-Postings zeigen sich weitere wichtige Merkmale, die Textformen in die internetbasierte Kommunikation einbringen: die flächige Präsentation von Information und die Möglichkeit zur multimodalen und hypertextuellen Gestaltung. Das von Ulrich Schmitz (2006, 2011) eingeführte Konzept der ,multimodalen Sehfläche‘ lässt sich sehr gut anwenden, um diese Gestaltungsmöglichkeiten $\mathrm{zu}$ beschreiben.

„Sichtbarkeit in der Fläche“ (Schmitz 2011, S. 30) ist Zeitlichkeit unter den Bedingungen von Textformen: Sie gewährleistet, dass alle Einheiten eines Kommunikats gleichzeitig - d.h. simultan - für die Rezipientinnen und Rezipienten präsent gemacht werden können (Fiehler 2005, S. 1182). Dabei wird

die temporale Logik linearer Kommunikation [...] durch eine räumliche Logik ersetzt, in der die Zusammenhänge zwischen Elementen nicht mehr eindimensional fortlaufend, sondern zweidimensional in der Fläche bestehen (Bucher 2011, S. 139).

Das ermöglicht die Rezeption in eigenem Tempo, in selbstgewählter Richtung und in beliebiger Wiederholung. Flächigkeit ermöglicht es, schriftliche Elemente räumlich zu komponieren und mit visuellen Mitteln der Graphostilistik und des Layouts zu arbeiten. Flächigkeit ermöglicht es, über parallele Kanäle zu kommunizieren und mehrere Sinne anzusprechen, wobei die Schrift gemeinsam mit anderen visuell basierten Zeichentypen erscheinen kann oder auch andere Zeichentypen gänzlich ohne Beteiligung von Schrift ein Kommunikat bilden können.

Die Textformen-basierte Kommunikation arbeitet mit multimodalen Sehflächen auf drei Ebenen:

- auf der Ebene des User Interfaces;

- auf der Ebene der Verlaufsprotokolle und hier als Gestaltungsleistung der zugrunde liegenden, die Kommunikation vermittelnden technischen Systeme;

- auf der Ebene des Inhalts einzelner Postings und damit als Leistung der jeweiligen Produzierenden und unter Nutzung von Assistenzfunktionen, die durch das technische System bereitgestellt werden. 


\subsection{Das Verlaufsprotokoll als multimodale Sehfläche}

Die von den Beteiligten übermittelten Beiträge werden für die Darstellung im Display vom System auf eine zweidimensionale Präsentationsfläche aufgetragen, die als endlose Schriftrolle vorgehalten wird (Abb. 6). Auf dem User Interface ist ein Fenster definiert, über das ein Ausschnitt aus dem Protokoll betrachtet werden kann; anhand eines Scrollbalkens kann diese Auswahl verändert und können frühere Teile des Protokolls in die Anzeige geholt werden.

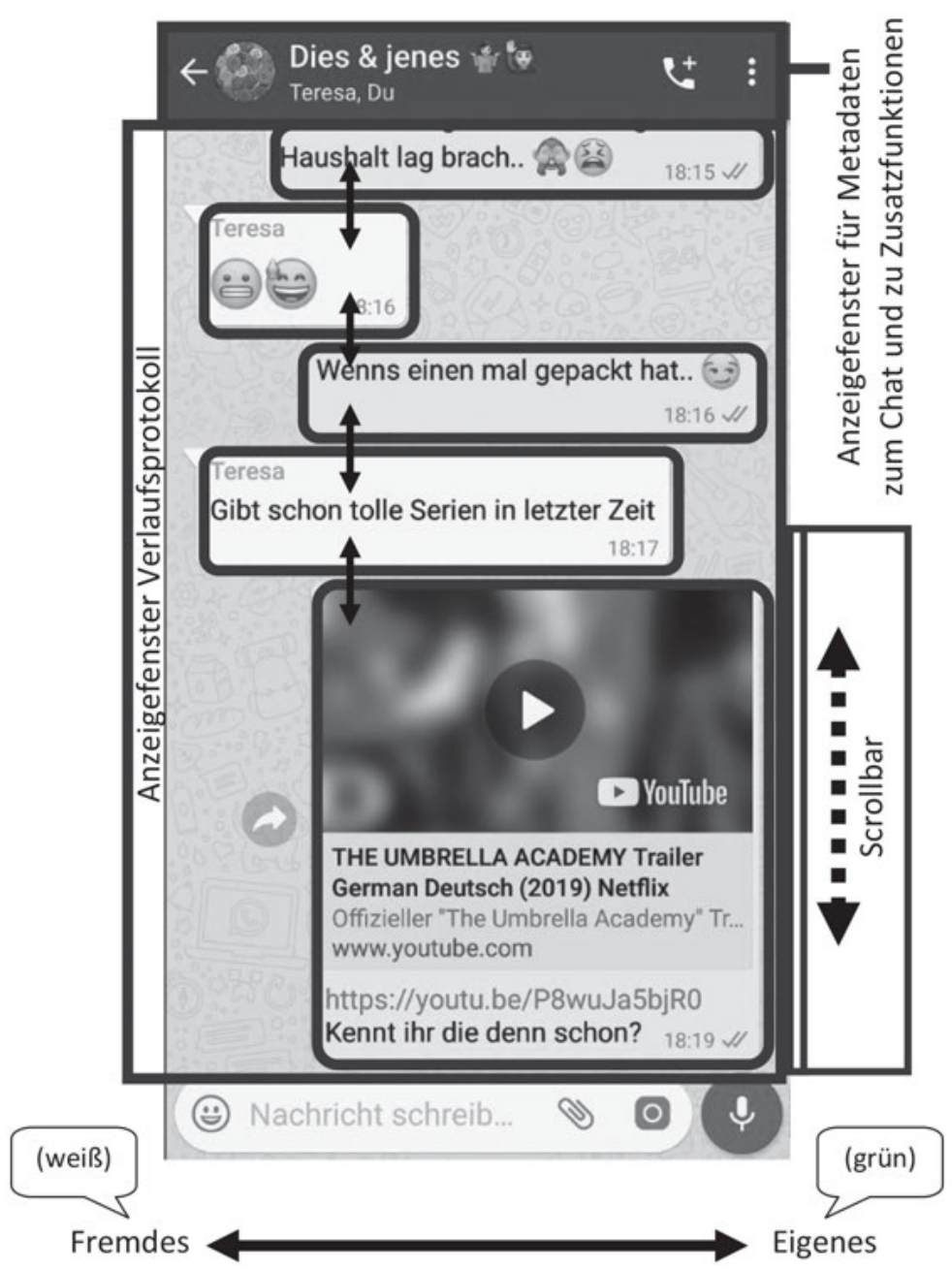

Abb. 6: Multimodale Gestaltung von User Interface und Verlaufsprotokoll am Beispiel WhatsApp 
Innerhalb des Protokolls sind die einzelnen Postings als Flächen mit klaren Begrenzungen ausgewiesen: Alles, was als user generated content zu einem Zeitpunkt von einem oder einer Beteiligten versendet wurde, wird innerhalb einer Parzelle dargestellt, die gegenüber dem Hintergrund farblich abgehoben ist. Dabei werden eigene Beiträge mit einer anderen Farbe hinterlegt als Beiträge der anderen Beteiligten. Eigenes ist rechtsbündig und Fremdes linksbündig an die vertikalen Begrenzungen der Protokollfläche angebunden. Als visuell-metaphorischer Verweis auf die Dialogizität des Austauschs fungiert die Sprechblasenoptik der hinterlegten Flächen.

\subsection{Das Posting als multimodale Sehfläche}

Die Gestaltung der Protokollfläche wird vom System verantwortet. Auf der Ebene der Postings liegt hingegen die Gestaltungshoheit bei den Nutzerinnen und Nutzern, die dazu auf Assistenzfunktionen der Software zurückgreifen können.

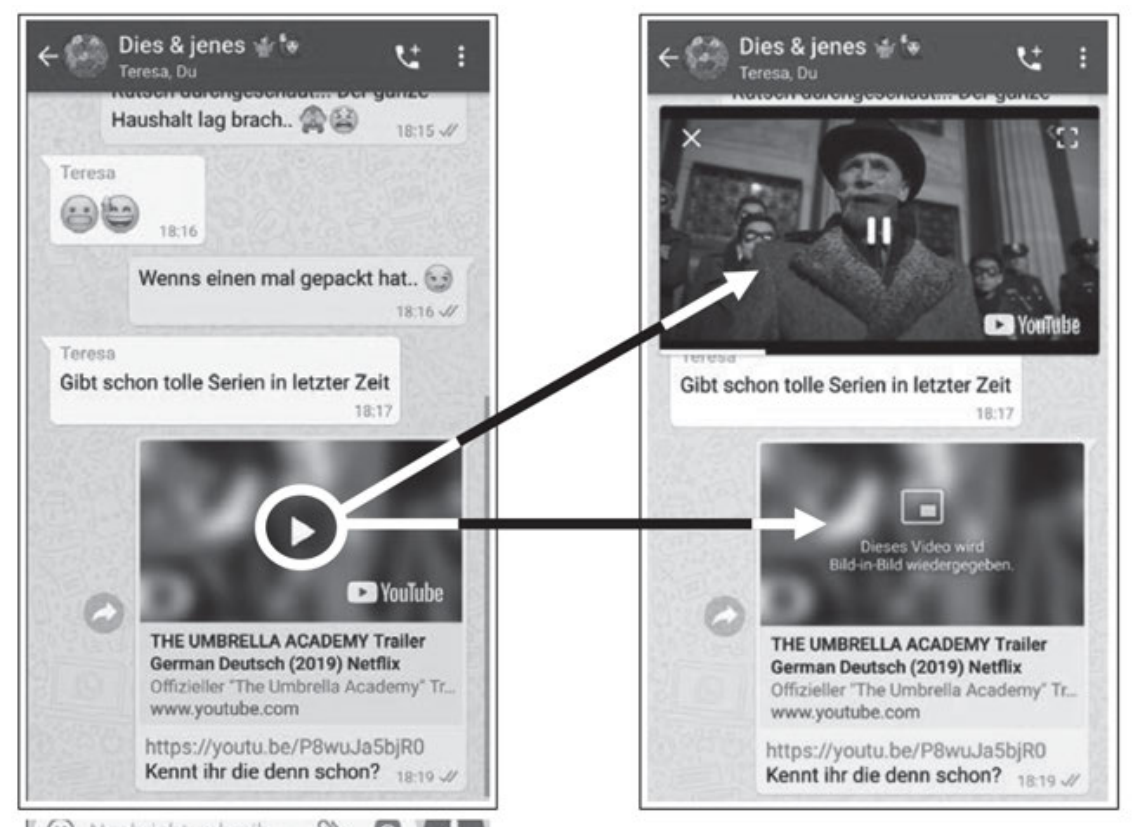

(:) Nachricht schreib... \& of 0

Abb. 7: Multimodale Gestaltung von Nutzerpostings und hypermediale Vernetzung mit externen Ressourcen (hier: mit einem YouTube-Video) 

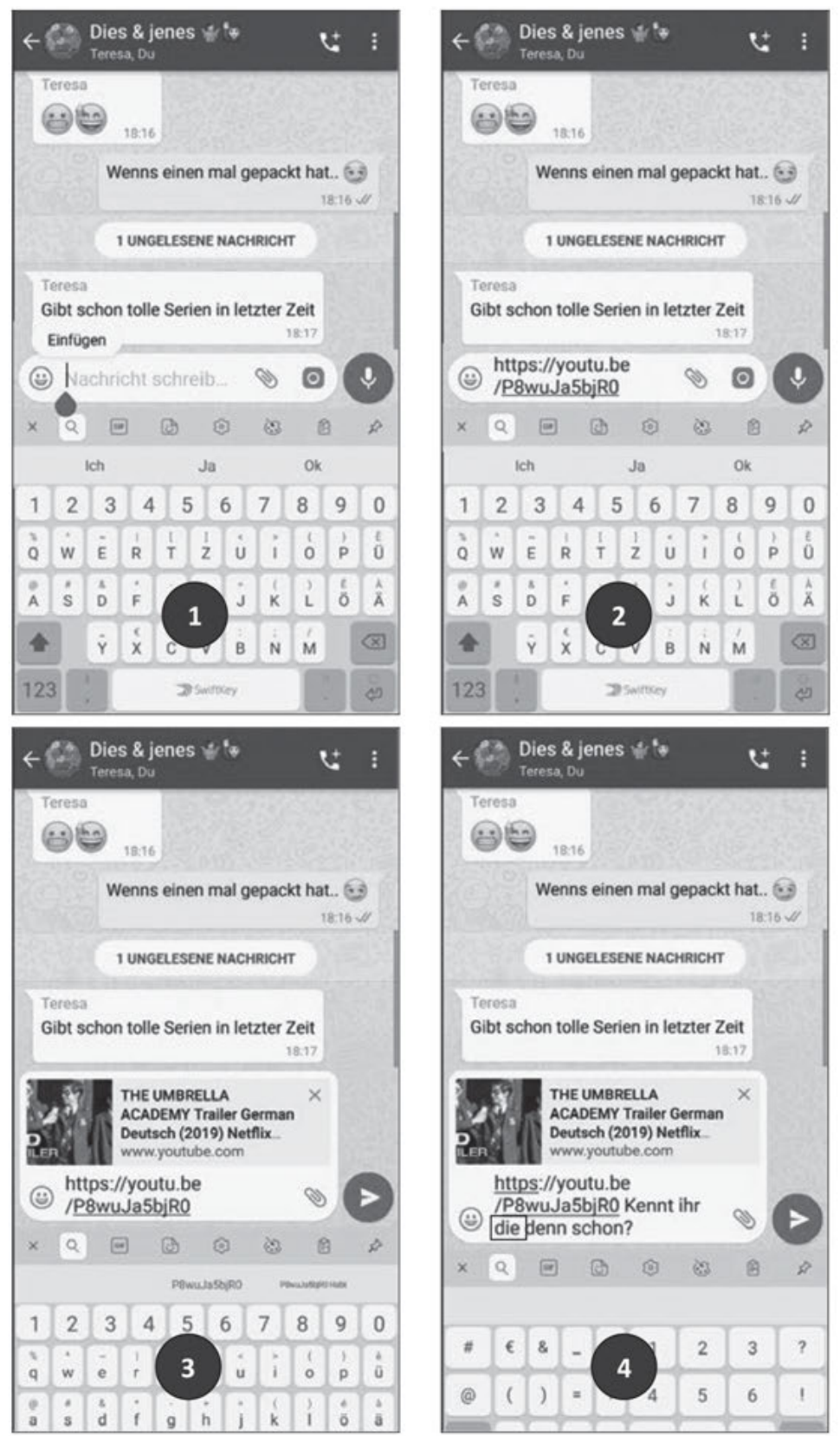

Abb. 8: Produktion des Nutzerpostings aus dem Beispiel in Abbildung 7 
Das letzte Posting im Beispiel aus Abbildung 6 enthält eine Vorschau auf ein YouTube-Video sowie, darauf deiktisch verweisend, den sprachlichen Äußerungsanteil „Kennt ihr die schon?“. Das Posting ist Teil einer Sequenz, in der sich die beiden Beteiligten über aktuelle TV-Serien unterhalten. Das Thema „Serien“ wurde im Vorgänger-Posting von Teresa sprachlich eingeführt. Die Vorschau auf das Video umfasst neben einem Screenshot verschiedene sprachlich präsentierte Angaben zur Serie, die vom System automatisch aus Metadaten zur entsprechenden Datei auf YouTube generiert wurden.

Die Vorschau auf das Video wird vom System als aktivierbares Bildschirmelement präsentiert (Abb. 7 links), das bei Berührung eine Bild-in-Bild-Wiedergabe auslöst, die einerseits sprachlich angezeigt wird („Dieses Video wird Bild-in-Bild wiedergegeben“) und andererseits als Layer, der über dem Protokoll eingeblendet wird, direkt rezipiert werden kann (Abb. 7 rechts). Auch die URL des YouTubeVideos, die vom Produzenten in sein Posting eingefügt wurde, wird vom System als Hyperlink und damit als aktivierbares Bildschirmelement dargestellt. Seine Aktivierung führt zum Aufruf der Zielseite im Webbrowser. Externe Ressourcen werden dadurch in die laufende Interaktion integrierbar.

Werfen wir abschließend noch einen Blick auf die Produktion des besprochenen Postings aus Abbildung 7. Die Analyse der Produktion, die in Abbildung 8 als Abfolge von vier Schritten veranschaulicht ist, macht sichtbar, wie stark das System selbst als künstlicher Akteur an der Gestaltung der Postings beteiligt ist.

$\mathrm{Zu}$ Beginn des Produktionsprozesses steht die Berührung des Eingabefelds mit dem Finger. Dadurch signalisiert die Nutzerin bzw. der Nutzer dem System, dass sie bzw. er einen Beitrag produzieren möchte. Das System blendet daraufhin eine Bildschirmtastatur in das Interface ein. Durch längere Berührung des Eingabefelds mit dem Finger wird eine Assistenzfunktion aktiviert, die es ermöglicht, Inhalte aus der Zwischenablage des Betriebssystems einzufügen. Das System blendet dazu eine interaktive Schaltfläche ein, die mit „Einfügen“ beschriftet ist (Abb. 8, Schritt 1). Der bzw. die Produzent/in tippt mit dem Finger auf diese Schaltfläche, wodurch eine zuvor in der Zwischenablage gespeicherte URL eingefügt wird, die auf das YouTube-Video verweist (Schritt 2).

Automatisch identifiziert das System daraufhin die eingegebene Zeichenfolge als URL, ruft im Hintergrund die Metadaten zur Videodatei aus YouTube ab und generiert eine Kurz-Vorschau (Schritt 3). Die Produzentin bzw. der Produzent weiß nun, dass er bzw. sie bestimmte Informationen zu der mit der URL referenzierten externen Ressource in seinem bzw. ihrem Beitrag nicht mehr sprachlich zu thematisieren braucht, sondern dass das System entsprechende Informationen automatisch in das Posting integriert. Er bzw. sie kann somit bei der weiteren Eingabe seines Beitrags (Schritt 4) bequem deiktisch auf die Zielressource verweisen, die 
über die Vorschau ad oculos verfügbar ist, wodurch das Verweisziel unmittelbar erschließbar wird. Bei der Betätigung der Senden-Funktion wird das Posting dann in ein für die Darstellung im Protokoll angepasstes Layout überführt und das Vorschaubild in ein abspielbares Medienobjekt transformiert (vgl. Abb. 7).

An diesem Beispiel zeigt sich mustergültig, wie technische Bedingungen der Kommunikation und der Beitragsgestaltung unmittelbar sprachliche Praktiken unterstützen und wie die sprachliche Gestaltung von Beiträgen durch technische Assistenzfunktionen mitgeprägt wird.

\subsection{Flächigkeit und Multimodalität als Ressourcen für Praktiken}

Die Präsentation und klare visuelle Abgrenzung der Postings auf der Protokollfläche sowie die Möglichkeit der multimodalen Gestaltung von Postings stellen Ressourcen dar, die die Interaktionsbeteiligten für die Interaktionsgestaltung nutzen. Exemplarisch sei dazu auf zwei aktuelle Untersuchungen verwiesen:

- Pragmatisierung der Sehfläche ,Verlaufsprotokoll‘: Androutsopoulos (2018, S. 728) erklärt die Nichtsetzung von Satzschlusszeichen in WhatsApp-Interaktionen als „Redundanz des Abschlusssignals durch die technologische Vermittlung: Die Betätigung der Enter-Taste und die grafisch-visuelle Rahmung der Einzelbeiträge [Hervorhebung MB] übernehmen im Grunde die abschlussmarkierende Leistung des Punktes in der digitalen Interaktion“. Die Setzung des Punktes wird damit frei für pragmatische Reinterpretation.

- Pragmatisierung der Sehfläche ,Posting‘: Beißwenger/Pappert (2019a, b) zeigen im Rahmen einer Untersuchung zum höflichen Handeln mit Emojis, wie positiv besetzte Emojis (z.B. das Daumen-hoch-Emoji) aufgrund ihrer Bildzeichenhaftigkeit als Modalisierer für gesichtsbedrohende Akte genutzt werden. Die Produzierenden machen sich dabei zunutze, dass Bildzeichen holistisch verarbeitet werden, ihre Bedeutung also nicht erst „erlesen“ werden muss, und eine individuelle Bedeutungszuschreibung auch unabhängig von der Kenntnis des sprachlichen Kontexts möglich ist. Mit dieser Eigenschaft können sie eingesetzt werden, um bei den Adressat/innen eine positive Erwartungshaltung in Bezug auf den Inhalt eines Postings auszulösen, noch bevor dieser Inhalt tatsächlich gelesen wurde. 


\section{Adaption des Konzepts der Textformen- basierten Interaktion für die Repräsentation von Korpora}

Während bei der linguistischen Modellierung der Erkenntniszugriff auf den Gegenstand und, als Konsequenz, die Wahl bestimmter theoretischer und methodischer Zugänge zu dessen Analyse im Vordergrund stehen, geht es bei der Repräsentation von Korpora um die Entwicklung von Modellen, die formal in einem Repräsentationsschema ausgedrückt werden und die der Korpusannotation zugrunde gelegt werden können. Speziell bei der kurationsgetriebenen Modellierung - das ist nach Jannidis (2017, S. 102) eine Modellierung, die auf eine langfristige und nachhaltige Bereitstellung von Forschungsressourcen angelegt ist - spielen dabei die Aspekte der Nachnutzbarkeit und der Interoperabilität eine wichtige Rolle. Nachnutzbarkeit beschreibt das Ziel, Ressourcen in Formaten vorzuhalten, die unabhängig von der Weiterentwicklung von Betriebssystemen und Spezialsoftware auch Jahrzehnte später mit Standardwerkzeugen verarbeitet und von Endnutzerinnen und -nutzern „verstanden“ werden können. Interoperabilität beschreibt das Ziel, Ressourcen so zu strukturieren, dass sie mit Ressourcen anderer Urheberinnen und Urheber kombiniert werden können. Eine wichtige Vorbedingung von Nachhaltigkeit und Interoperabilität bilden Repräsentations-Standards. Standards dienen nach Henning Lobin dazu, „dass nicht jeder das Rad neu erfinden muss“ (Lobin 2010, S. 107): Sie ermöglichen, dass für die zu lösende Aufgabe auf Lösungen zurückgegriffen werden kann, die sich in anderen Projekten ähnlicher Art bereits bewährt haben. Ein wichtiger Baustein, um Standards zu etablieren, ist die Entwicklung geeigneter Datenmodelle.

Die Repräsentation von Korpora ist nicht primär eine „technische“ Aufgabe, auch wenn die Annotationen, mit denen auf der Grundlage eines Datenmodells die Daten in einem Korpus angereichert werden, für ungeschulte Augen auf den ersten Blick sehr technisch anmuten mögen. Gerade die linguistische Perspektive wird bei der Entwicklung von Modellen für die Strukturrepräsentation von Korpusdaten dringend benötigt. Mit Jannidis (2017) kann Modellierung als der Punkt betrachtet werden, „wo das geisteswissenschaftliche Verständnis eines Weltausschnitts und die Kompetenz zur formalen Modellierung zusammentreffen und im besten Fall neue Fragestellungen und neue Forschungen ermöglichen“ (ebd., S. 107f.).

Das Konzept der Textformen-basierten Interaktion liefert gute Argumente dafür, weshalb Modelle für die Strukturannotation von redigierten Texten und von transkribierten Gesprächen nicht 1:1 geeignet sind, Korpora internetbasierter Kommunikation zu beschreiben. Auch bei der Repräsentation von Korpora erweist 
sich die internetbasierte Kommunikation als etwas Drittes, das aber in den Traditionen von Interaktion und Text verwurzelt ist. Etablierte Standards für die Strukturannotation texttragender Objekte in den Geisteswissenschaften, wie sie von der Text Encoding Initiative (TEI) angeboten werden, umfassen bislang keine Modelle für Sprachdaten internetbasierter Kommunikation (vgl. Lüngen/Kupietz i.d.Bd.).

In den vergangenen Jahren hat eine Arbeitsgruppe mit Vertreterinnen und Vertretern von Korpusprojekten zu mehreren europäischen Sprachen ein Schema für die Annotation von Korpora internetbasierter Kommunikation entwickelt, das auf die Encoding-Formate der TEI aufsetzt und diese erweitert. Das Schema baut auf das TEI-Modell für strukturierte Texte auf. Gespeicherte Verlaufsprotokolle werden dabei zunächst als strukturierte Dokumente betrachtet, die Absatzgliederungen, Mittel der Textgestaltung, Medienobjekte und Hyperlinks enthalten können. Für diese textgliedernden und textgestaltenden Mittel stehen im TEI-Standard ausgearbeitete Modelle zur Verfügung, die für die internetbasierte Kommunikation nur geringfügig angepasst werden müssen. Zentral - und neu ist Folgendes: Die Mittel der Textgliederung und -gestaltung werden im Schema für die internetbasierte Kommunikation um ein übergeordnetes, neues Modellierungskonstrukt erweitert, in dessen Rahmen sie auftreten können. Dieses übergeordnete Konstrukt trägt den Namen <post>. Posts haben Produktcharakter. Sie reisen durchs Netz als kleine Dokumente mit nutzergeneriertem Inhalt und zugeordneten Metadaten. Ihre Funktion ist es, einen Beitrag zu einer laufenden Interaktion zu leisten, deshalb hat jede Instanz des Konzepts Post eine/n eigene/n Urheber/in. Posts werden nach ihrer Übermittlung als flächige Gliederungseinheiten in einem kontinuierlich fortgeschriebenen Dokument präsentiert. In dieser Form bilden sie die Verarbeitungsgrundlage für die anderen Interaktionsbeteiligten (Veranschaulichung des Modells: Abb. 9).

Oberhalb der Ebene der Posts gibt es ein Strukturelement, das die Bündelung von Abfolgen von Post-Instanzen zu Verlaufsprotokollen beschreibt. Hierfür ist im Modell ein neuer Subtyp des im TEI-Standard vorhandenen Elements < div> (division für Textgliederungen) definiert. Damit sind die beiden für die internetbasierte Kommunikation prägenden Textformen - das Posting und das Verlaufsprotokoll - als Strukturelemente verfügbar. Das Modell ist in Form eines RNGSchemas beschrieben, das über das TEI-Wiki ${ }^{3}$ zur Verfügung gestellt wird und das für Bedarfe eigener Projekte erweitert und modifiziert werden kann. Eine ausführliche Begründung und Darstellung des Modells bietet Beißwenger (2018).

3 https://wiki.tei-c.org/index.php?title=SIG:CMC (Stand: 18.7.2019). 


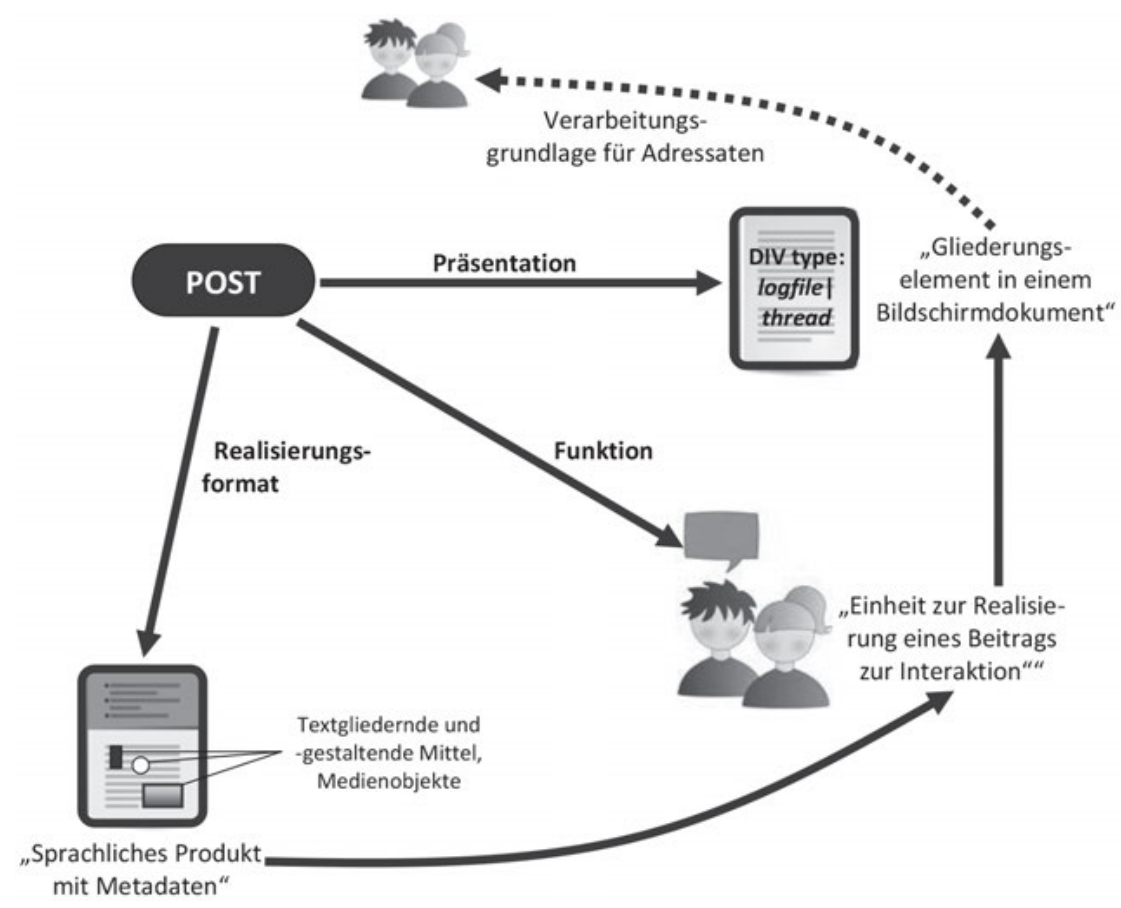

Abb. 9: Modell <post> im TEl-Repräsentationsschema für Korpora internetbasierter Kommunikation

\section{Fazit}

Das in diesem Beitrag vorgeschlagene Konzept der Textformen-basierten Interaktion bietet einen Beschreibungsrahmen sowohl für die linguistische Analyse internetbasierter Kommunikation als auch für die Repräsentation entsprechender Daten in Korpora.

Für die linguistische Analyse ergibt sich die Möglichkeit, die Perspektive der Interaktion und die Perspektive der Analyse von Textformen systematisch miteinander zu verknüpfen. Interaktion ist das Ziel, Textformen sind das Mittel der Kommunikation. Interaktion wird durch Textformen zeitlich zerdehnt. Daraus ergeben sich Konsequenzen für die Sequenzorganisation und für die Gestaltung von Beiträgen. Die Zeitlichkeitsbedingungen des Gesprächs stehen nicht zur Verfügung. Die Integration der Perspektiven von Text und Schreiben ermöglicht es, die neuen Formen der Interaktion als das darstellbar zu machen, was sie sind: eine Weiterentwicklung sequenziell intendierten sprachlichen Handelns unter den Bedingungen von Visualität, Persistenz, Flächigkeit und Multimodalität. 
Adaptiert für den Bereich Korpusrepräsentation ermöglicht es das Modell, Standards für die Repräsentation von textuellen Artefakten, wie sie die Text Encoding Initiative (TEI) bereitstellt, für die Strukturannotation von Korpora internetbasierter Kommunikation anzupassen und um Modellierungskonstrukte zu erweitern, die die Indienstnahme von Textformen für die Zwecke der Interaktion darstellbar machen. Für die Entwicklung von Annotationsschemata und - mittelfristig - die mögliche Etablierung eines entsprechenden Annotationsstandards muss damit nichts grundlegend Neues entwickelt werden; auch hier erweist sich die Adaption des Instrumentariums, das für die Analyse von Textformen bereits vorhanden ist, als geeignetes Vorgehen, um auf der Basis des Bekannten das tatsächlich Neue und Innovative an der internetbasierten Kommunikation zu beschreiben und in Korpora abzubilden. Unter der in diesem Beitrag zugrunde gelegten Perspektive, die internetbasierte Kommunikation als ein Drittes auf einem Entwicklungsweg von Organisationsformen sprachlichen Handelns aufzufassen, der von der mündlichen, direkten Interaktion über die gesellschaftliche Aneignung und Ausdifferenzierung von Textformen und Texttraditionen hin zu einer Indienstnahme von Textformen für Zwecke der vermittelten Interaktion führt, ist ein solches Vorgehen nicht nur praktikabel, sondern - mag man dem vorgeschlagenen Modell folgen - auch in hohem Maße informativ.

\section{Literatur}

Androutsopoulos, Jannis K. (2018): Digitale Interpunktion: Stilistische Ressourcen und soziolinguistischer Wandel in der informellen digitalen Schriftlichkeit von Jugendlichen. In: Ziegler, Arne (Hg.): Jugendsprachen. Aktuelle Perspektiven internationaler Forschung/ Youth languagages. Current perspectives of international research. Teilbd. 1. Berlin u.a., S. 721-748.

Auer, Peter (2000): On line-Syntax - oder: was es bedeuten könnte, die Zeitlichkeit der mündlichen Sprache ernst zu nehmen. In: Sprache und Literatur 85, S. 43-56.

Auer, Peter (2007): Syntax als Prozess. In: Hausendorf (Hg.), S. 95-124.

Beißwenger, Michael (2003): Sprachhandlungskoordination im Chat. In: Zeitschrift für germanistische Linguistik 31, 2, S. 198-231.

Beißwenger, Michael (2005): Interaktionsmanagement in Chat und Diskurs. Technologiebedingte Besonderheiten bei der Aushandlung und Realisierung kommunikativer Züge in Chat-Umgebungen. In: Beißwenger, Michael/Storrer, Angelika (Hg.): Chat-Kommunikation in Beruf, Bildung und Medien: Konzepte - Werkzeuge - Anwendungsfelder. Stuttgart, S. 63-87.

Beißwenger, Michael (2007): Sprachhandlungskoordination in der Chat-Kommunikation. (= Linguistik - Impulse \& Tendenzen 26). Berlin u.a.

Beißwenger, Michael (2016): Praktiken in der internetbasierten Kommunikation. In: Deppermann, Arnulf/Feilke, Helmuth/Linke, Angelika (Hg.): Sprachliche und kommunikative Praktiken. (= Jahrbuch des Instituts für Deutsche Sprache 2015). Berlin u.a., S. 279-310. 
Beißwenger, Michael (2018): Internetbasierte Kommunikation und Korpuslinguistik: Repräsentation basaler Interaktionsformate in TEI. In: Lobin, Henning/Schneider, Roman/Witt, Andreas (Hg.): Digitale Infrastrukturen für die germanistische Forschung. (= Germanistische Sprachwissenschaft um 2020 6). Berlin u.a., S. 307-349.

Beißwenger, Michael/Pappert, Steffen (2019a): How to be polite with emojis: a pragmatic analysis of face work strategies in an online learning environment. In: European Journal for Applied Linguistics 7, 2, S. 225-253.

Beißwenger, Michael/Pappert, Steffen (2019b): Face work mit Emojis. Was linguistische Analysen zum Verständnis sprachlichen Handelns in digitalen Lernumgebungen beitragen können. In: Beißwenger, Michael/Knopp, Matthias (Hg.): Soziale Medien in Schule und Hochschule: Linguistische, sprach- und mediendidaktische Perspektiven. (= Forum Angewandte Linguistik 63). Frankfurt a.M., S. 101-143. Internet: www.peterlang.com/ view/9783631791622/9783631791622.00008.xml (Stand: 4.10.2019).

Bucher, Hans-Jürgen (2011): Multimodales Verstehen oder Rezeption als Interaktion. Theoretische und empirische Grundlagen einer systematischen Analyse der Multimodalität. In: Diekmannshenke, Hajo/Klemm, Michael/Stöckl, Hartmut (Hg.): Bildlinguistik. Theorie - Methoden - Fallbeispiele. (= Philologische Studien und Quellen 228). Berlin, S.123-156.

Couper-Kuhlen, Elisabeth (2007): Prosodische Prospektion und Retrospektion im Gespräch. In: Hausendorf (Hg.), S. 69-94.

Deppermann, Arnulf (2007): Grammatik und Semantik aus gesprächsanalytischer Sicht. (= Linguistik - Impulse \& Tendenzen 14). Berlin u.a.

Deppermann, Arnulf (2018): Sprache in der multimodalen Interaktion. In: Deppermann, Arnulf/ Reineke, Silke (Hg.): Sprache im kommunikativen, interaktiven und kulturellen Kontext. (= Germanistische Sprachwissenschaft um 2020 3). Berlin u.a., S. 51-85.

Dürscheid, Christa (2005): Medien, Kommunikationsformen, kommunikative Gattungen. In: Linguistik online 22, 1, S. 3-16. Internet: https://bop.unibe.ch/linguistik-online/article/ view/752/1284 (Stand: 30.4.2019)

Dürscheid, Christa/Brommer, Sarah (2009): Getippte Dialoge in Neuen Medien. Sprachkritische Aspekte und linguistische Analysen. In: Linguistik Online 37, 1, S. 3-20. Internet: https:// bop.unibe.ch/linguistik-online/article/view/511/851 (Stand: 30.4.2019).

Ehlich, Konrad (1983): Text und sprachliches Handeln. Die Entstehung von Texten aus dem Bedürfnis nach Überlieferung. In: Assmann, Aleida/Assmann, Jan/Hardmeier, Christof (Hg.): Schrift und Gedächtnis. Beiträge zur Archäologie der literarischen Kommunikation. (= Archäologie der literarischen Kommunikation 1). München, S. 24-43.

Ehlich, Konrad (1984): Zum Textbegriff. In: Rothkegel, Annely/Sandig, Barbara (Hg.): Text Textsorten - Semantik. Linguistische Modelle und maschinelle Verfahren. Hamburg, S. 531-550.

Fiehler, Reinhard (2005): Gesprochene Sprache. In: Duden: Der Duden in zwölf Bänden. Bd. 4: Die Grammatik. Unentbehrlich für richtiges Deutsch. 7., vlg. neu erarb. u. erw. Aufl. Mannheim u.a., S. 1175-1256.

Garcia, Angela C./Baker Jacobs, Jennifer (1999): The eyes of the beholder: Understanding the turn-taking system in quasi-synchronous computer-mediated communication. In: Research on Language and Social Interaction 32, 4, S. 337-367.

Hausendorf, Heiko (2007): Die Prozessualität des Gesprächs als Dreh- und Angelpunkt der linguistischen Gesprächsforschung. In: Hausendorf (Hg.), S. 11-32.

Hausendorf, Heiko (Hg.) (2007): Gespräch als Prozess. Linguistische Aspekte der Zeitlichkeit verbaler Interaktion. (= Studien zur Deutschen Sprache 37). Tübingen. 
Hausendorf, Heiko/Lindemann, Katrin/Ruoss, Emanuel/Weinzinger, Caroline (2015): Ko-Konstruktionen in der Schrift? Zur Unterscheidung von face-to-face-Interaktion und Textkommunikation am Beispiel des Editierens fremder Beiträge in einem Online-Lernforum. In: Dausendschön-Gay, Ulrich/Gülich, Elisabeth/Krafft, Ulrich (Hg.): Ko-Konstruktionen in der Interaktion. Die gemeinsame Arbeit an Äußerungen und anderen sozialen Ereignissen. Bielefeld, S. 111-138.

Hoffmann, Ludger (2004): Chat und Thema. In: Beißwenger, Michael/Hoffmann, Ludger/ Storrer, Angelika (Hg.): Internetbasierte Kommunikation. (= Osnabrücker Beiträge zur Sprachtheorie 68). Oldenburg, S. 103-122.

Imo, Wolfgang (2013): Sprache in Interaktion. Analysemethoden und Untersuchungsfelder. (= Linguistik - Impulse \& Tendenzen 49). Berlin u.a.

Imo, Wolfgang (2015): Vom Happen zum Häppchen ... Die Präferenz für inkrementelle Äußerungsproduktion in internetbasierten Messengerdiensten. (= Networx 69). Hannover. Internet: www.mediensprache.net/networx/networx-69.pdf (Stand: 1.5.2019).

Imo, Wolfgang/Lanwer, Jens (2019): Interaktionale Linguistik. Eine Einführung. Stuttgart. Jannidis, Fotis (2017): Grundlagen der Datenmodellierung. In: Fotis, Jannidis/Kohle, Hubertus/ Rehbein, Malte (Hg.): Digital Humanities. Eine Einführung. Stuttgart, S. 99-108.

König, Katharina/Hector, Tim M. (2017): Zur Theatralität von WhatsApp-Sprachnachrichten. Nutzungskontexte von Audio-Postings in der mobilen Messenger-Kommunikation. (= Networx 79). Internet: www.mediensprache.net/networx/networx-79.pdf (Stand: 1.5.2019).

Liedtke, Frank (2009): Schrift und Zeit. Anmerkungen zu einer Pragmatik des Schriftgebrauchs. In: Birk, Elisabeth/Schneider, Jan G. (Hg.): Philosophie der Schrift. (= Germanistische Linguistik 285). Tübingen, S. 75-94.

Lobin, Henning (2010): Computerlinguistik und Texttechnologie. (= Linguistik für Bachelor 3282). Paderborn.

Schmitz, Ulrich (2006): Schriftbildschirme. Tertiäre Schriftlichkeit im World Wide Web. In: Androutsopoulos, Jannis K./Runkehl, Jens/Schlobinski, Peter/Siever, Torsten (Hg.): Neuere Entwicklungen in der linguistischen Internetforschung. (= Germanistische Linguistik 186/187). Hildesheim, S. 184-208.

Schmitz, Ulrich (2011): Sehflächenforschung. Eine Einführung. In: Diekmannshenke, Hajo/ Klemm, Michael/Stöckl, Hartmut (Hg.): Bildlinguistik. Theorien - Methoden - Fallbeispiele. (= Philologische Studien und Quellen 228). Berlin, S. 23-42.

Selting, Margaret/Couper-Kuhlen, Elizabeth (2000): Argumente für die Entwicklung einer ,interaktionalen Linguistik؛ In: Gesprächsforschung - Online-Zeitschrift zur verbalen Interaktion 1, S. 76-95. Internet: http://gespraechsforschung-online.de/heft2000/ ga-selting.pdf (Stand: 1.5.2019).

Storrer, Angelika (2013): Sprachstil und Sprachvariation in sozialen Netzwerken. In: Frank-Job, Barbara/Mehler, Alexander/Sutter, Tilmann (Hg.): Die Dynamik sozialer und sprachlicher Netzwerke. Konzepte, Methoden und empirische Untersuchungen an Beispielen des WwW. Wiesbaden, S. 331-366.

Storrer, Angelika (2014): Sprachverfall durch internetbasierte Kommunikation? Linguistische Erklärungsansätze - empirische Befunde. In: Plewnia, Albert/Witt, Andreas (Hg.): Sprachverfall? Dynamik - Wandel - Variation. (= Jahrbuch des Instituts für Deutsche Sprache 2013). Berlin u.a., S. 171-196.

Weingarten, Rüdiger (1989): Die Verkabelung der Sprache. Grenzen der Technisierung von Kommunikation. (= Fischer alternativ: Perspektiven 4181). Frankfurt a.M.

Zitzen, Michaela/Stein, Dieter (2005): Chat and conversation: A case of transmedial stability? In: Linguistics 42, 5, S. 983-1021. 
